MKG-Chirurg 2020 $13: 269-275$

https://doi.org/10.1007/s12285-020-00271-5

Online publiziert: 8. Oktober 2020

(c) Der/die Autor(en) 2020

\section{Redaktion}

S. Haßfeld, Dortmund

\section{S. Meister ${ }^{1}$ S. Haßfeld ${ }^{2} \cdot$ A. Burmann ${ }^{1}$}

'Abteilung „HealthCare", Fraunhofer-Institut für Software- und Systemtechnik ISST, Dortmund, Deutschland

${ }^{2}$ Klinik für Mund-, Kiefer- und Gesichtschirurgie - Plastische Operationen, Klinikum Dortmund Nord, Dortmund, Deutschland

\title{
Digitalisierung in der Mund-, Kiefer- und Gesichtschirurgie
}

\section{Vom Status quo zur Vision}

Das Bundesministerium für Gesundheit hat in den letzten Monaten eine Vielzahl von Gesetzen zur Förderung der Digitalisierung im Gesundheitswesen erlassen. Das Terminservice- und Versorgungsgesetz (TSVG), das Digitale-Versorgung-Gesetz (DVG) und das Patientendaten-Schutz-Gesetz (PDSG) fordern technische, organisatorische und finanzielle Strukturen ein. So ist die Umsetzung der elektronischen Patientenakte (ePA) nach $\$ 291$ a SGB V mittlerweile terminiert - ein wichtiger Schritt weg von analogen Kommunikationswegen und hin $\mathrm{zu}$ einer digitalen Kommunikation. $\mathrm{Zu}$ letzt hatte eine Analyse errechnen wollen, dass durch die Digitalisierung im Gesundheitswesen bis zu 34 Mrd. € eingespart werden könnten [1].

Die schon heute umgesetzten digitalen Versorgungsketten bewegen sich zwischen Bedürfnissen und Erwartungen, aber auch Enttäuschungen, denn die Zielbilder der Digitalisierung aus Sicht der Kostenträger, Ärztinnen und Ärzte sowie Patientinnen und Patienten sind nicht immer komplementär [2]: Die Patientinnen und Patienten erwartet die bestmögliche Versorgung mit einem hohen Personalisierungsgrad, Leistungserbringerinnen und Leistungserbringer erwünschen eine Entlastung bei dokumentarischen Prozessen sowie Steigerung der Effektivität, und Kostenträger fordern mehr Effizienz.

Die Schaffung einer durchgängigen und für alle Akteurinnen und Akteure gleichermaßen wertschöpfenden Digitalisierung setzt einen einheitlichen Digitalisierungstand voraus. Diese Analyse setzt sich aus diesem Grund mit dem Status quo in der Mund-, Kiefer- und Gesichts(MKG)-Chirurgie auseinander. Hierzu wurden der internationale Stand mithilfe einer strukturierten Literaturanalyse erhoben sowie qualitative Interviews mit Expertinnen und Experten geführt.

\section{Digitalisierung und digitale Reife}

Wissenschaft und Wirtschaft haben zuletzt den Begriff der Industrie 4.0 geprägt, in der digitale Produkte und Innovationen prädominierend sind [3]. Die 4.0Familie hat schnell Zuwachs aus dem Gesundheitswesen erhalten: Begriffe wie Gesundheit 4.0 oder Krankenhaus 4.0 stellen die Annahme auf, dass die bestehenden Herausforderungen, wie der demografische Wandel oder der Fachkräftemangel, faktisch durch Digitalisierung lösbar sind [4]. Die begriffliche Genese zur Digitalisierung im Gesundheitswesen kann auf nunmehr 40 Jahre historische Entwicklung verweisen, wie - Abb. 1 zeigt. Die definitorischen Nuancen dahinter sind jedoch zumeist marginal.

Durch das DVG sowie das PDSG wurde maßgeblich der Begriff der digitalen Gesundheitsanwendung (DiGa) geprägt. Ärztinnen und Ärzte können diese gemäß der Digitalen-Gesundheitsanwendungen-Verordnung (DiGaV) erstattungsfähig verordnen. Jedoch sind sie auch aufgefordert, den Schutz der in der Telematikinfrastruktur zu verarbeitenden Patientendaten mit sicherzustellen und Versicherte bei der Verwaltung ihrer ePA zu unterstützen.

Digitalisierung ist keine einmalige Aktivität, sondern ein Transformationsprozess und erwartet von jedem Leistungserbringer und jeder Leistungserbringerin, basierend auf seinem bzw. ihrem individuellen Digitalisierungsstand, die technischen und organisatorischen Voraussetzungen $\mathrm{zu}$ schaffen. Hierzu gilt es, den aktuellen Stand genau beschreiben zu können - man spricht vom sog. digitalen Reifegrad. Modelle wie das internationale „electronic medical report adoption model“ (EMRAM) der Healthcare Information and Management Systems Society (HIMSS) helfen, das Maß der Vernetzung von ITSystemen zu quantifizieren [5]. Sie vernachlässigen jedoch eine der kritischsten Komponenten: den Faktor Mensch. Die Autoren forschen deshalb mit ihrem „digital health maturity model“ [6] an einem mehrdimensionalem Modell (• Abb. 2).

Mithilfe dieses Modells wurde in Kooperation mit der Krankenhausgesellschaft Nordrhein-Westfalen (KGNW) der Status quo der Digitalisierung der 344 Mitgliedshäuser erhoben [7, 8]. Ebenso erfolgte die bundesweite Erhebung zur digitalen Reife in Kooperation mit dem Clinotel Krankenhausverbund gGmbH, vertretend für über 60 Krankenhäuser. Das Modell bildet die Basis für die nachfolgende Erhebung in der MKGChirurgie, die mit einer strukturierten Erhebung der Literatur beginnt. 


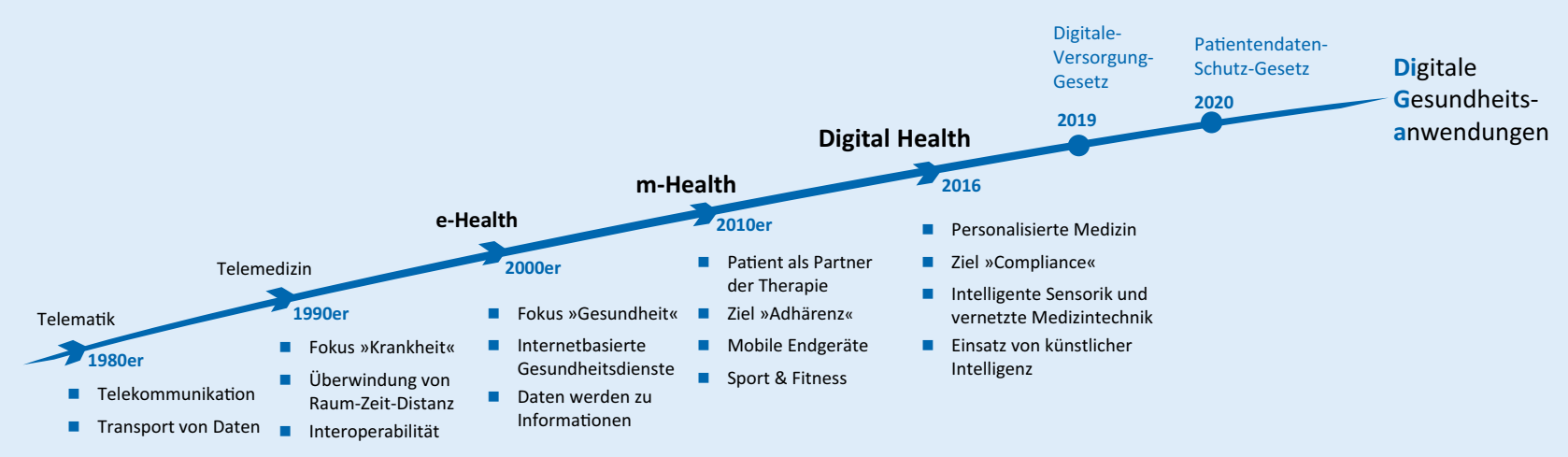

Abb. 1 ॥ Entwicklung von der Telemedizin zu digitalen Gesundheitsanwendungen (DiGa). (Mit freundl. Genehmigung $\odot$ S. Meister (Fraunhofer ISST), alle Rechte vorbehalten)

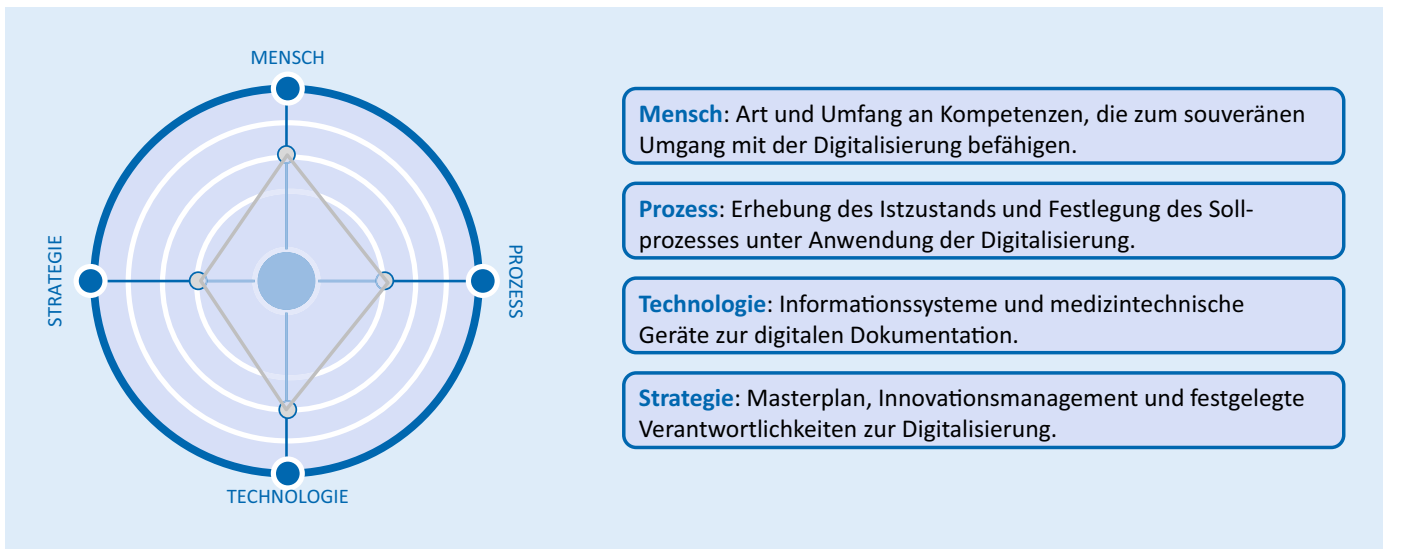

Abb. $2 \triangleleft$ Mehrdimensionales "digital health maturity model" unter Berücksichtigung des Faktors Mensch. (Mit freundl. Genehmigung $\odot$ S. Meister (Fraunhofer ISST), alle Rechte vorbehalten)

\section{Strukturierte Literatur- analyse zur Erfassung des internationalen Status quo}

Zu Beginn möchten die Autoren dem Begriff der Digitalisierung für den Bereich der MKG-Chirurgie einen Rahmen geben. Art und Umfang des internationalen Einsatzes von digitaler Bildgebung, Telemedizin, Apps oder auch künstliche Intelligenz (KI) in diesem Bereich sollen hierzu mithilfe externer Literatur erhoben werden.

\section{Material und Methoden}

Die strukturierte Literaturanalyse (SLA) wurde entlang der methodischen Vorgaben von Kitchenham und Charters [9] sowie Kitchenham et al. [10] durchgeführt.Zur Recherche wurde das etablierte Programm „Publish or Perish“ [11] eingesetzt, ein Softwareprogramm, das akademische Zitate aus einer Vielzahl von Datenquellen abruft und analysiert. Wir verwendeten die Option der Stichwortsuche, was bedeutet, dass „Publish or Perish" versuchen wird, Suchbegriffe überall in den resultierenden Publikationen zu finden. Es wurde der folgende Suchterm genutzt: („maxillofacial surgery“) AND (digital OR telemedicine OR „artificial intelligence“ OR „digital imaging“). Die Anzahl der abgerufenen Einträge wurde auf 100/Anfrage begrenzt. Danach wurden die Ergebnismenge gefiltert und nichtwissenschaftliche Einträge wie Patente oder Websites entfernt. Die Ergebnismenge wurde jeweils nach Zahl der Zitate, Hirsch(h)-Index, Datum und Ranking sortiert, und es wurden jeweils die Top-3-Publikationen in die Betrachtung einbezogen.

\section{Ergebnis}

Die radiologische Bildgebung ist einer der am umfassendsten digitalisierten Bereiche der Medizin und auch in der historischen Betrachtung Vorreiterin im
Bereich der Adaptation von Innovation, Definition von Branchenstandards und elektronischen Archivierungssystemen [12]. Der Fachbereich der MKG-Chirurgie ist mit diesem Fach eng vernetzt und profitiert daher von diesen Entwicklungen. Darüber hinaus entwickeln sich auf Basis dessen fachspezifische Anwendungen wie z.B. die digitale Volumentomographie, die ihrerseits erfolgreich in der Domäne eingesetzt wird, und deren Vorteile zunehmend auch über den Fachbereich hinaus genutzt werden [13]. Einsatzgebiete im Bereich der MKG-Chirurgie sind v.a. Diagnostik, aber auch Eingriffs- und Implantatplanung [14]. Weiterhin zu nennen ist die Anwendung von computergestützter Navigation in MKG-chirurgischen Eingriffen, wobei die reale Operation mit digitalen Bilddaten und Navigationsinformationen überlagert wird. Letztgenannte Systeme wirken sich sowohl auf das medizinische Ergebnis als auch dafür notwendige zeitliche Ressourcen positiv aus. Bezeichnend 
ist, dass die Zahl der Veröffentlichungen für digitale Systeme in den genannten Bereichen zusammengefasst bereits $a b$ 2002 stark zunimmt und die Klimax im Jahr $2014 \mathrm{zu}$ finden ist [15-17].

Über die weite Verbreitung und Etablierung solcher punktuellen Systeme hinaus wird die Integration von digitalen Systemen in einen Prozess über Systemgrenzen einzelner Akteurinnen und Akteure z. B. im Bereich der Herstellung von Implantaten durch 3-D-Druck beschrieben [18]. Darüber hinaus wird die digitale Unterstützung der „patient journey“über die Grenzen einzelner Leistungserbringerinnen und Leistungserbringer hinweg nur am Rande thematisiert. Farman et al. diskutieren Effekte wie Patientendatensicherheit begleitend zu ihrer allgemeinen Betrachtung des Einflusses der Digitalisierung auf die Zahnheilkunde und kommen zu dem Schluss, dass digitale Bildgebung und Volumendarstellung das Mindestmaß der klinischen Ausstattung sein sollten. Die Aspekte Effizienz und wirtschaftlicher Nutzen sind ebenso Teil des wissenschaftlichen Interesses: Joda und Brägger [19] kommen in einem Vergleich von ImplantatprothesenWorkflows an 20 Patientinnen und Patienten in der Schweiz zu dem Ergebnis, dass bei einer digitalen WorkflowUnterstützung mit einer Effizienzsteigerung und geringeren direkten Kosten im Vergleich zu einem gänzlich analogen Ablauf zu rechnen ist. Anzumerken ist hierzu, dass der Unterscheidungsgegenstand eben eine digitale Bildgebung und Planung des Implantats im Gegensatz zu einem Realmodell war. Der Grad der prozessualen Durchgängigkeit und Integration ebensolcher Systeme über Grenzen von Anbietern hinweg wird für den Bereich Zahnheilkunde in einigen Artikeln aufgegriffen, explizit für die MKG-Chirurgie aber noch nicht betrachtet $[20,21]$.

\section{》) Veröffentlichungen über digitale Systeme nehmen im Bereich der MKG-Chirurgie seit 2002 stark zu}

Aziz et al. [22] setzten sich bereits 2009 mit den Möglichkeiten des Einsatzes von
Telemedizin zu Konsultation, Kommunikation sowie Behandlungsplanung in der MKG-Chirurgie auseinander. Witzke und Specht [23] konkretisierten diese Arbeit durch Möglichkeiten zur Telekonsultation mithilfe von „mobile health“. Den Fokus von fortschrittlichen Systemen auf die Akteurinnen und Akteure, also Patientinnen und Patienten sowie Mitarbeiterinnen und Mitarbeiter, bei Digitalisierungsbestrebungen zu richten und die Integration von Daten entlang einer ganzheitlichen Versorgungskette $\mathrm{zu}$ betrachten, ist auch für den Bereich MKGChirurgie Teil der aktuellen und zukünftigen Ausgestaltung. Zwar wird konstatiert, dass die KI derzeit noch unterrepräsentiert ist, jedoch ein hohes Potenzial für die Zukunft der MKG-Chirurgie besitzt, z.B. zur Behandlung von Dysgnathien [24].

\section{Experteninterviews}

Die Erfassung des Status quo der Digitalisierung in Deutschland sowie die Benennung der Chancen und Risiken erfolgten durch die Befragung von Expertinnen und Experten. Im Fokus standen die Dimensionen Mensch und Technologie.

\section{Material und Methoden}

Es wurde sich für einen qualitativen Forschungsansatz entschieden, in dem semistrukturierte Interviews mit Expertinnen und Experten aus verschiedenen Forschungsbereichen durchgeführt wurden. Der verwendete methodische Rahmen umfasst 5 Schritte, die sich an der $\mathrm{Pu}$ blikation von Dicicco-Bloom und Crabtree [25] orientieren. Als Stichprobenverfahren für die Befragten wurde sich für die Benennung von Schlüsselinformanten im Sinne von Expertinnen und Experten (mindestens einem Vertreter) wie folgt entschieden [26, 27]:

1. Klinikdirektor im Krankenhaus $(\mathrm{KH})$, männlich (m), weiblich (w), divers (d), >20 Jahre Berufserfahrung,

2. Facharzt, KH m, w, d, <10 Jahre Berufserfahrung,

3. Praxisleitung, $\mathrm{m}, \mathrm{w}, \mathrm{d},>10$ Jahre Berufserfahrung,
Hier steht eine Anzeige. Me Springer 
4. angestellter Facharzt, Praxis, m, w, d, $<10$ Jahre Berufserfahrung,

5. medizinisch-technische Fachkraft, m, w, d, > 10 Jahre Berufserfahrung,

6. medizinisch-technische Fachkraft, m, w, d, <10 Jahre Berufserfahrung.

Die Rekrutierung erfolgte unterstützt durch Prof. Dr. Dr. Stefan Haßfeld sowie den Sprecher des Bündnis Junge Ärzte, Max Tischler. Es konnten 8 Expertinnen und Experten gewonnen werden.

Der Fragebogen wurde in 2 Teile aufgeteilt, jeder auf einer eigenen Seite, um das Kriterium der Pseudonymisierung zu erfüllen. Die erste Seite umfasste Basisdaten wie Name, Beruf, Position und eine zufällige Kennung. Die zweite Seite enthielt die gleiche Kennung und folgende offenen Fragen:

- F1: Was sind die häufigsten Indikationen, mit welchen eine Patientin bzw. ein Patient bei Ihnen vorstellig wird?

- F2: Bitte beschreiben Sie den grundsätzlichen MKG-Prozess in 2-3 verschiedenen Szenarien: Wann wird eine Patientin bzw. ein Patient bei Ihnen vorstellig, wer hat vorher welche Daten bereits zu ihr oder ihm erhoben, welche Schritte werden in Ihrer Praxis durchgeführt, und welcher nachgelagerten Versorgung wird die Patientin bzw. der Patient anschließend zugeführt?

- F3: Mit welchen (professionellen und privaten) Akteurinnen und Akteuren tauschen Sie im Arbeitsalltag (Patienten-)Daten oder Informationen aus?

- F4: Was sind die größten Herausforderungen Ihres Fachbereichs, externer, aber auch interner Natur?

- F5: Bitte beschreiben Sie Aspekte/ Teilbereiche Ihrer Arbeit, in welchen Ihr Fachbereich (in den letzten 5 bis 10 Jahren) durch die Digitalisierung verändert wurde.

- F6: Was sind, wenn Sie an den eigenen Arbeitsalltag, aber auch den Ihrer Mitarbeitenden, denken, Bereiche, in denen Sie sich Erleichterungen durch Digitalisierung erhoffen würden?

- F7: Was sind Bereiche, in denen Sie Bedenken gegenüber der Digitalisierung haben?

MKG-Chirurg 2020 - 13:269-275 https://doi.org/10.1007/s12285-020-00271-5

(c) Der/die Autor(en) 2020

\section{S. Meister · S. Haßfeld · A. Burmann}

\section{Digitalisierung in der Mund-, Kiefer- und Gesichtschirurgie. Vom Status quo zur Vision}

\section{Zusammenfassung}

Hintergrund. Mit dem Digitale-VersorgungGesetz (DVG) und dem PatientendatenSchutz-Gesetz (PDSG) wurden Gesetze zur Stärkung einer digital unterstützten Gesundheitsversorgung erlassen. Digitalisierung beansprucht, die Effektivität und Effizienz im Sinne der Leistungserbringerinnen und Leistungserbringer und Patientinnen und Patienten stärken zu können. Sie erfordert jedoch auch, Prozesse und Verantwortlichkeiten neu zu strukturieren, in Technologie zu investieren und digitale Kompetenzen sicherzustellen.

Fragestellung. Welche Chancen und Risiken ergeben sich durch Digitalisierung aus Sicht der Akteurinnen und Akteure der Mund-, Kiefer- und Gesichts(MKG)-Chirurgie, und welcher Status quo ergibt sich im internationalen Vergleich?

Material und Methode. Anwendung von Experteninterviews $(n=8)$ als qualitatives Erhebungsinstrument und Durchführung einer strukturierten Literaturanalyse.
Ergebnisse. Digitalisierung hilft, Therapien zu optimieren $(n=7)$, wobei die digitale Bildgebung ein geschätztes Instrument $(n=8)$ in der MKG-Chirurgie ist. Es fehlen jedoch Angebote zur Aneignung digitaler Kompetenzen $(n=6)$, und zudem werden die Anforderungen des Datenschutzes $(n=4)$ kritisch gesehen.

Schlussfolgerung. Digitalisierung erfordert gerade zu Beginn hohe zeitliche und auch finanzielle Investitionen. Zudem sind neue Kompetenzen erforderlich, um Souveränität im Umgang mit digitalen Produkten sicherstellen zu können. Es wird angestrebt, die qualitativen Ergebnisse nachfolgend durch eine quantitative Analyse zu verifizieren.

\section{Schlüsselwörter}

Gesundheitsversorgung · Patientensicherheit . Digitale Kompetenz · Digitale Reife · Umfragen und Fragebogen

\section{Digitalization in oral and maxillofacial surgery. From status quo to vision}

Abstract

Background. With the Digital Care Act (DVG) and the Patient Data Protection Act (PDSG) laws were passed to strengthen digitally supported healthcare. Digitalization claims to be able to strengthen effectiveness and efficiency in the sense of healthcare providers and patients; however, digitalization also requires restructuring processes and responsibilities, investing in technology and ensuring digital skills.

Objective. What opportunities and risks result from digitalization from the perspective of the players in the field of oral and maxillofacial surgery and what is the status quo in international comparison?

Material and methods. Expert interviews $(n=8)$ were applied as a qualitative survey instrument and a structured literature analysis was carried out.
Results. Digitalization helps to optimize treatment $(n=7)$, whereby digital imaging is an appreciated instrument $(n=8)$ in oral and maxillofacial surgery; however, there is a lack of offers for the acquisition of digital skills $(n=6)$ and, in addition, the requirements of data protection $(n=4)$ are viewed critically. Conclusion. Especially at the beginning, digitalization requires high investments in terms of time and money. In addition, new skills are required to ensure sovereignty in handling digital products. It is intended to subsequently verify the qualitative results by means of a quantitative analysis.

\section{Keywords}

Health services - Patient safety - Digital competence - Digital maturity · Surveys and questionnaires 


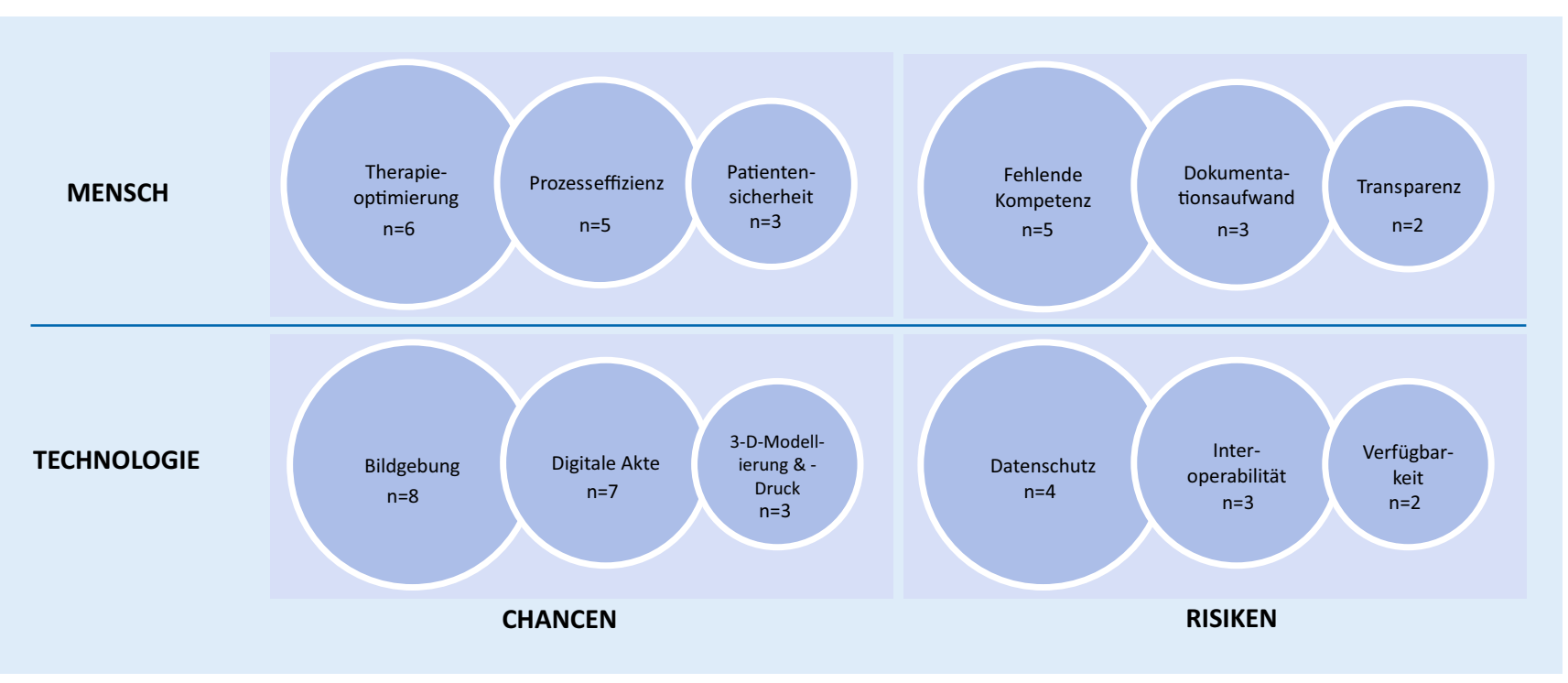

Abb. 3 ॥ Zusammengefasste Darstellung der Ergebnisse $(n=8)$, kodiert nach Digitalisierung aus Sicht von Mensch und Technologie, aufgetragen gegen die Chancen und Risiken. Dargestellt sind die jeweils 3 häufigsten Nennungen. (Mit freundl. Genehmigung $\odot$ S. Meister [Fraunhofer ISST], alle Rechte vorbehalten)

- F8: Wenn Sie an die mittel- und langfristige Zukunft (5 und mehr Jahre) denken: „Was sind Szenarien/ Bereiche, in denen Sie sich Innovationen durch Digitalisierung für Ihren Fachbereich erhoffen?"

Die Durchführung der Interviews erfolgte telefonisch. Es wurde mit der Erläuterung des Zwecks des Interviews begonnen. Im Folgenden werden das Format und die Art des Interviews beschrieben, d.h. (1) der Interviewer bzw. die Interviewerin stellt zunächst eine Frage, (2) gibt danach eine kurze Erklärung $\mathrm{ab}$ und (3) gibt der interviewten Person Gelegenheit, Verständnisfragen zu stellen. Die Dauer des Interviews (30 min) wurde der interviewten Person mitgeteilt. Zur Sicherung des Datenschutzes werden (1) die von der befragten Person zur Verfügung gestellten Informationen geschützt und (2) der Fragebogen pseudonymisiert. Der bzw. die Interviewte wurde über die Möglichkeiten zur Kontaktaufnahme mit den Interviewenden informiert. Das Transkript des Interviews wurde der interviewten Person zu Abnahme und Freigabe zur Verfügung gestellt. Für die Analyse der Interviewdaten entschieden sich die $\mathrm{Au}$ toren für eine Kombination von offener Kodierung zusammen mit axialer Kodierung, wie sie von Wiesche et al. [28] vorgeschlagen wurde. Die Überarbeitung der gesammelten Daten stützte sich auf die Verwendung von Kodes, um Textsegmente zu markieren und dann Textsegmente mit ähnlichem Inhalt zu gruppieren. Die Kodierung erfolgte mit der Zielsetzung der Klassifikation von Aussagen nach Mensch, Technologie, Chancen und Risiken.

\section{Ergebnis}

Die Ergebnisse der kodierten Transkripte sind in - Abb. 3 dargestellt. Es werden jeweils die 3 häufigsten Chancen und Risiken der Digitalisierung aus Sicht von Technologie und Mensch visualisiert.

Die Befragten bescheinigen der Digitalisierung, die Effizienz von Prozessen zu erhöhen $(n=5)$. Daten sind zu jeder Zeit an einem Ort verfügbar und der Bearbeitungsstand nachvollziehbar, sodass Leistungserbringerinnen und Leistungserbringer entlastet werden können. Das Verfügbarmachen von Daten erlaubt es zudem, Therapien z.B. durch einen höheren Grad der Personalisierung zu optimieren $(n=6)$. Prozesstransparenz und eine optimierte Therapie besitzen aus Sicht der Expertinnen und Experten das Potenzial, die Patientensicherheit zu erhöhen $(n=3)$.

Fehlende digitale Kompetenzen stellen aus Sicht der Befragten das größte Ri- siko dar $(n=5)$, denn nur durch eine spezifische Ausbildung kann Entlastung geschaffen werden. Fehlende Kompetenzen können $\mathrm{zu}$ Unsicherheiten führen und schlimmstenfalls die Patientensicherheit durch fehlerhafte Bedienung gefährden $(n=2)$. Der Chance der gestiegenen Prozesseffizienz steht aus Sicht der Expertinnen und Experten aber eine höhere Transparenz gegenüber $(n=2)$. Transparenz bedeutet, dass das Handeln der Ärztin bzw. des Arztes gläsern wird und dieses auch für eine unerlaubte Leistungsbeurteilung missbraucht werden könnte. Ebenso besteht das Gefühl, dass Digitalisierung trotz der erwarteten Prozesseffizienz gerade für die medizinischen Leistungserbringer $\mathrm{zu}$ einem erhöhten Dokumentationsaufwand führt $(n=3)$.

》D Der bereits weit verbreiteten
digitalen Bildgebung wird eine
besonders hohe Bedeutung
beigemessen

Aus Sicht der technologischen Chancen wird der bereits weit verbreiteten digitalen Bildgebung eine besonders hohe Bedeutung beigemessen $(n=8)$. Die Bilddaten sind direkt verfügbar, und weitergehende bildanalytische Software erlaubt eine Verbesserung der Eingriffsplanung 
und -durchführung. Eine institutionsinterne, digitale Akte ist nach Meinung der Expertinnen und Experten sowohl für Praxen wie auch für Kliniken eine notwendige Voraussetzung $(n=7)$ und wird bereits bei 7 Expertinnen und Experten eingesetzt. Mehrheitlich $(n=5)$ wird konstatiert, dass der intersektorale, institutionsübergreifende Datenaustausch derzeit - wenn überhaupt - nur im Fall von Bilddaten erfolgt. Möglichkeiten zur digitalen Eingriffsplanung, Modellierung und Simulation sowie des 3D-Drucks stellen insbesondere im Bereich der Rekonstruktion eine Verbesserung dar $(n=3)$. Perspektivisch werden an dieser Stelle auch Verfahren der KI gesehen, um aus Daten basale Modelle zu erlernen $(n=2)$.

Den Chancen stehen jedoch auch Risiken gegenüber, insbesondere das Risiko des Datenschutzes $(n=4)$ sowie des hiermit steigenden Aufwands zur Einhaltung. Beherrschung und Sicherstellung von Datenschutz und Datensicherheit fordern insbesondere die MKG-Praxen $(n=3)$. Eng hiermit verknüpft sind die Notwendigkeit eines ausfallsicheren Betriebs sowie die daraus resultierende Abhängigkeit von der Technologie, aber auch zu externen Dienstleistern $(n=3)$.

Alle $(n=8)$ befragten Expertinnen und Experten haben bereits an verschiedenen Kliniken und/oder Praxen gearbeitet, woraus sich ein sehr breites Bild des Durchdringens der Digitalisierung ergibt. Zwar besteht in jedem Krankenhaus mit dem Krankenhausinformationssystem ein basales IT-System zur Dokumentation, jedoch ist dieses entlang des Patientenprozesses zumeist nur schlecht mit anderen Systemen vernetzt $(n=3)$. Die Dokumentation in Krankenhäusern ist zumeist $(n=3)$ durch die Ärztinnen und Ärzte eigenständig zu erbringen. In modernen MKG-Praxen ist der gesamte Workflow digital unterstützt, und die Dokumentation wird maßgeblich durch das Praxispersonal durchgeführt $(n=2)$. Sowohl für Krankenhäuser wie auch Praxen ergibt sich die Herausforderung der finanziellen Investition $(n=6)$, wobei Praxen keinem internen Wettbewerb mit anderen Fachabteilungen unterliegen und somit eine zielgerichtete Investition möglich ist.

\section{Diskussion}

Die Bildgebung war und ist seit jeher Taktgeber für digitale Innovationen in der Medizin, und dies zeigt sich auch im Bereich der MKG-Chirurgie. Die Expertinnen und Experten bestätigen, dass sich hierdurch Arbeitserleichterungen sowie Möglichkeiten der Therapieverbesserung ergeben. International zeichnet sich ein ähnliches Bild, wodurch sich schon früh wesentliche digitale Strukturen ausprägen konnten. An dieser Stelle muss jedoch auch kritisch betrachtet werden, dass sich derzeit fernab der Bildgebung nur langsam digital unterstützte Prozesse innerhalb der MKG-Chirurgie ausprägen. Dies betrifft sowohl die Unterstützung administrativer Prozesse, die intersektorale Kommunikation mit anderen Versorgern als auch die Einbindung der Patientinnen und Patienten z. B. über telemedizinische Apps.

\section{》) Aus- und Weiterbildung adressieren Kompetenzen zum Umgang mit digitalen Technologien bislang zu wenig}

Kurzfristig muss das Risiko der fehlenden digitalen Kompetenz in den Fokus genommen werden. Aus- und Weiterbildung adressieren notwendige Kompetenzen zum souveränen Umgang mit digitalen Technologien nur wenig. Weder die Curricula der medizinischen Ausbildung noch nachgelagerte Weiterbildungsprogramme unterstützen bisher in relevanter Weise den Kompetenzerwerb, um den Anforderungen des DVG sowie der Forderung des Einsatzes von DiGa gerecht werden zu können.

Die Befragung der Expertinnen und Experten und die strukturierte Literaturanalyse verstehen sich als Startpunkt und nicht als abgeschlossene, vollständige Erhebung zu Art und Umfang der Digitalisierung in der MKG-Chirurgie. Die Autoren streben deshalb an, eine quantitative Analyse über einen Online-Survey anzuknüpfen.

\section{Fazit für die Praxis}

- Bereits heute sind digitale Bildgebung, 3-D-Eingriffsplanung sowie digitale Dokumentation verbreitete Aspekte der Digitalisierung.

- Zwischen MKG-Praxen und MKGKliniken sind Unterschiede bei der Art und dem Umfang der Digitalisierung erkennbar.

- Für den Menschen in seiner Rolle als leistungserbringende Person ergeben sich Chancen zu Therapieoptimierung und Prozesseffizienz durch eine bessere Datenverfügbarkeit. Jedoch werden zum souveränen Umgang mit der Digitalisierung derzeit im Curriculum nicht vermittelte digitale Kompetenzen benötigt.

- Für Patientinnen und Patienten besteht das Potenzial einer erhöhten Patientensicherheit sowie der Personalisierung von therapeutischen Verfahren z. B. bei der Rekonstruktion und somit einer zu erwartenden, höheren Lebensqualität.

\section{Korrespondenzadresse}

Dr. S. Meister
Abteilung „HealthCare“,
Fraunhofer-Institut für
Software- und Systemtechnik
ISST
Emil-Figge-Str. 91,
44227 Dortmund,
Deutschland
sven.meister@
isst.fraunhofer.de




\section{Einhaltung ethischer Richtlinien}

Interessenkonflikt. S. Meister, S. Haßfeld und A. Burmann geben an, dass kein Interessenkonflikt besteht.

Für diesen Beitrag wurden von den Autoren keine Studien an Menschen oder Tieren durchgeführt. Für die aufgeführten Studien gelten die jeweils dort angegebenen ethischen Richtlinien. Von allen an der Befragung Beteiligten liegt eine schriftliche Einverständniserklärung vor. Die Zustimmung einer Ethikkommission war nicht notwendig.

Open Access. Dieser Artikel wird unter der Creative Commons Namensnennung 4.0 International Lizenz veröffentlicht, welche die Nutzung, Vervielfältigung, Bearbeitung, Verbreitung und Wiedergabe in jeglichem Medium und Format erlaubt, sofern Sie den/die ursprünglichen Autor(en) und die Quelle ordnungsgemäß nennen, einen Link zur Creative Commons Lizenz beifügen und angeben, ob Änderungen vorgenommen wurden.

Die in diesem Artikel enthaltenen Bilder und sonstiges Drittmaterial unterliegen ebenfalls der genannten Creative Commons Lizenz, sofern sich aus der Abbildungslegende nichts anderes ergibt. Sofern das betreffende Material nicht unter der genannten Creative Commons Lizenz steht und die betreffende Handlung nicht nach gesetzlichen Vorschriften erlaubt ist, ist für die oben aufgeführten Weiterverwendungen des Materials die Einwilligung des jeweiligen Rechteinhabers einzuholen.

Weitere Details zur Lizenz entnehmen Sie bitte der Lizenzinformation auf http://creativecommons.org/ licenses/by/4.0/deed.de.

\section{Literatur}

1. Hehner S, Biesdorf S, Möller M (2018) Digitalisierung im Gesundheitswesen: die Chancen für Deutschland. McKinsey \& Company, Düsseldorf. https://www.mckinsey.de/ /media/mckinsey/ locations/europe\%20and\%20middle\%20east/ deutschland/news/presse/2018/2018-09-25digitalisierung\%20im\%20gesundheitswesen/ mckinsey92018digitalisierung\%20im\%20ge sundheitswesendownload.ashx. Zugegriffen: 02.06.2020

2. Meister S (2019) Das neue Gesundheits-Ich souverän, selbstbestimmt und digital unterstützt? G+GWissenschaft 19(3):7-14

3. Bauernhansl T (2014) Die Vierte Industrielle Revolution - Der Weg in ein wertschaffendes Produktionsparadigma. In: Bauernhansl T, ten Hompel M, Vogel-Heuser B (Hrsg) Industrie 4.0 in Produktion, Automatisierung und Logistik. Springer Vieweg, Wiesbaden, S5-35

4. Meister S, Becker S, Leppert F et al (2017) Digital Health, Mobile Health und Co. - Wertschöpfung durch Digitalisierung und Datenverarbeitung. In: Pfannstiel MA, Da-Cruz P, Mehlich H (Hrsg) Digitale Transformation von Dienstleistungen im Gesundheitswesen I. Springer, Wiesbaden, S 185-212

5. HIMMS Analytics (2017) Electronic medical record adoption model. http://www.himss.eu/ healthcare-providers/emram. Zugegriffen: 12. März2018
6. Burmann A, Deiters W, Meister S (2019) Digital Health Maturity Index. In:Pfannstiel MA, Da-CruzP, Mehlich $\mathrm{H}$ (Hrsg) Digitale Transformation von Dienstleistungen im Gesundheitswesen VI. Gabler, Wiesbaden, S3-18

7. Fischer B, Burmann A, Brinkkötter N et al (2019) Das Digitale Krankenhaus. Krankenhausgesellschaft NRW, Düsseldorf. https://www.das-digitalekrankenhaus.nrw/wp-content/uploads/2020/05/ Das_digitale_Krankenhaus_KGNW-Whitepaper2_final.pdf.Zugegriffen:02.06.2020

8. Meister S (2019) Das "Digitale Krankenhaus": Reifegradmessung zur Standortbestimmung. Krankenhaus-IT J 3:40-41

9. Kitchenham B, Charters S (2007) Guidelines for performing systematic literature reviews in software engineering

10. Kitchenham B, Pearl Brereton O, Budgen D et al (2009) Systematic literature reviews in software engineering - A systematic literature review. Inf Softw Technol 51(1):7-15

11. Harzing AW (2007) Publish or perish. http://www. harzing.com/pop.htm. Zugegriffen:02.06.2020

12. Kim N, Choi J, Yi J et al (2013) An engineering view on megatrends in radiology: digitization to quantitative tools of medicine. Korean J Radiol 14(2):139-153

13. de Vos W, Casselman J, Swennen GRJ (2009) Conebeam computerized tomography (CBCT) imaging of the oral and maxillofacial region: a systematic review of the literature. Int J Oral Maxillofac Surg 38(6):609-625

14. Swennen GRJ, Mollemans W, Schutyser F (2009) Three-dimensional treatment planning of orthognathic surgery in the era of virtual imaging. J Oral Maxillofac Surg 67(10):2080-2092

15. Ewers R, Schicho K, Undt G et al (2005) Basic research and 12 years of clinical experience in computer-assisted navigation technology: a review. Int J Oral Maxillofac Surg 34(1):1-8

16. Farman AG, Levato CM, Gane D et al (2008) In practice: how going digital will affect the dental office. J Am Dent Assoc 139(Suppl):14S-19S

17. Guerrero ME, Jacobs R, Loubele M et al (2006) State-of-the-art on cone beam CT imaging for preoperative planning of implant placement. Clin Oral Investig 10(1):1-7

18. Tack P, Victor J, Gemmel P et al (2016) 3D-printing techniques in a medical setting: a systematic literature review. BioMed Eng OnLine 15(1):115

19. Joda T, Brägger U (2015) Digital vs. conventional implant prosthetic workflows: a cost/time analysis. Clin Oral Implants Res 26(12):1430-1435

20. Joda T,ZaroneF,FerrariM(2017)The completedigital workflow in fixed prosthodontics: a systematic review. BMCOral Health 17(1):124

21. Patel N (2010) Integrating three-dimensional digital technologies for comprehensive implant dentistry. JAm Dent Assoc 141(Suppl 2):20S-24S

22. Aziz SR, Ziccardi VB (2009) Telemedicine using smartphones for oral and maxillofacial surgery consultation, communication, and treatment planning. JOral Maxillofac Surg 67(11):2505-2509

23. Witzke K, Specht O (2017) M-health telemedicine and telepresence in oral and maxillofacial surgery. Int JReliab Qual E-Healthcare 6(4):37-48

24. Bouletreau P, Makaremi M, Ibrahim B et al (2019) Artificial intelligence: applications in orthognathic surgery. J Stomatol Oral Maxillofac Surg 120(4):347-354

25. Dicicco-BloomB, CrabtreeBF (2006) The qualitative research interview. Med Educ 40(4):314-321
26. Coyne IT (1997) Sampling in qualitative research. Purposeful and theoretical sampling; merging or clear boundaries? J Adv Nurs 26(3):623-630

27. Marshall MN (1996) Sampling for qualitative research. Fam Pract 13(6):522-525

28. Wiesche M, Jurisch MC, Yetton PW et al (2017) Grounded theory methodology in information systems research. MISQ 41(3):685-701 